\title{
EDITORIAL
}

\section{Nobel Prize to immunology}

\author{
This year's Nobel Prize to Ralph Steinman, Jules Hoffmann and Bruce Beutler marked the \\ significance of the advances made over the past 4 decades in the field of innate immunology.
}

The announcement of this year's Nobel Prize in Physiology or Medicine was bitter-sweet for the world of immunology. The excitement about the Prize being awarded to the immunologists Ralph Steinman, Jules Hoffmann and Bruce Beutler was overshadowed by the sad news that Ralph Steinman had passed away 3 days before the announcement by the Nobel Assembly.

Ralph Steinman (Rockefeller University, New York, USA) was awarded one half of the Nobel Prize "for his discovery of the dendritic cell and its role in adaptive immunity". Nowadays, the central role of dendritic cells in sensing pathogen infection, priming $\mathrm{T}$ cell responses and shaping adaptive immune responses through cytokine production is indisputable. Back in 1973, however, when Ralph Steinman described "a novel cell type ... that can assume a variety of branching forms, and constantly extend and retract many fine cell processes"1, the scientific community was doubtful about the significance of these findings. "It was a bit like having two Popes - it was the dendritic cells versus the macrophages", as Siamon Gordon, University of Oxford, UK, recalls in Nature (Nature News, 3 Oct 2011). Since then, the work of Ralph Steinman and numerous other investigators has unravelled the function of dendritic cells as professional antigen presenters that shape adaptive immune responses, leading to immune reactivity or tolerance (for an overview of the function of dendritic cells, see our recent Poster).

Nothing is more convincing for demonstrating the value of a scientific discovery than the successful application of the new findings to the treatment of disease. The significance of the work of Ralph Steinman is highlighted by the fact that several dendritic cell-based cancer vacthis year's Nobel Prize highlights the importance of the initial discoveries that brought the roles of dendritic cells and TLRs into focus onosed with pancreatic cancer 4 years ago, was him treated with an experimental dendritic cell-based therapy.

The discoveries of the other two Nobel laureates have equally big potential for the development of immunebased therapies. Jules Hoffman (French National Centre for Scientific Research, Institute of Cell and Molecular other half of this year's Nobel Prize for their discoveries concerning the activation of innate immunity.

In 1996, Jules Hoffmann and his colleagues, primed by the parallels between immune signalling pathways in mammals and the embryonic development-associated Toll signalling pathway in Drosophila melanogaster, discovered the essential role of the cell surface receptor Toll in innate immune responses against bacteria and fungi in fruit flies ${ }^{3}$. The description of a role for Toll in pathogen recognition triggered an explosion in the field of innate immunity, which at that time was puzzled by how pathogen-derived signals activate innate immune cells and thus lead to the effective priming of adaptive immune responses.

One year after the publication of Hoffmann's Cell paper, the first human Toll-like receptor (TLR) gene now known as TLR4- was cloned by Metzhitov and Janeway ${ }^{4}$. Their work, published in Nature, showed that overexpression of a constitutively active human TLR resulted in the expression of cytokines and costimulatory molecules. Some months later, in September 1998, Godowski and colleagues reported in Nature that lipopolysaccharide (LPS)-induced gene expression depends on TLR2 signalling 5 .

These key events prepared the ground for the finding by Beutler and colleagues published in December 1998 that LPS is the ligand for TLR4, as mutations in the Tlr4 gene in mice were found to result in resistance to LPSinduced sepsis ${ }^{6}$. It is remarkable that, since the elucidation of the role of TLRs in pathogen sensing, over 15,000 publications have focused on their immune function. In the past years, the main players in TLR signalling have been identified and numerous TLR-binding pathogenassociated and damage-associated molecular patterns have been characterized. Furthermore, synthetic TLR agonists are being tested in clinical trials for their therapeutic potential in inflammatory diseases.

In this new era of immunology, this year's Nobel Prize highlights the importance of the initial discoveries that brought the roles of dendritic cells and TLRs into focus. Biology, Strasbourg, France) and Bruce Beutler (Scripps Research Institute, La Jolla, California, USA) share the

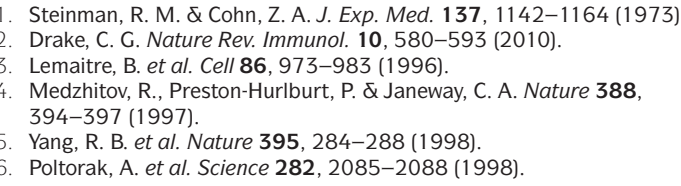

